\title{
Computing with Complex Optical Networks
}

\author{
Kan $\mathrm{Wu}^{1}$, Behrad Gholipour, ${ }^{1}$ Wenchao $\mathrm{Hu}^{1}$, Perry Ping Shum ${ }^{1}$, Nikolay Zheludev ${ }^{1,2}$ \\ and Cesare Soci ${ }^{1}$
}

\begin{abstract}
Using simple fiber networks for proof-of-principle demonstrations, we give examples of natural computing in linear optical networks, like solving polynomial (P) and nondeterministic polynomial (NP) problems, and in nonlinear optical networks, like metaheuristic optimization and neuromorphic computing.
\end{abstract}

\section{INTRODUCTION}

Natural computing encompasses nature-inspired problem-solving techniques and new computing paradigms based on natural materials. So far both numerical and physical platforms have been exploited to develop natural computation, including evolutionary algorithms, neural networks, quantum and DNA computing, as well as neuromorphic electronic chips. Optical computing has been explored to certain extent, but primarily in the free space, with strict requirements on alignment, collimation and detection of the optical signals, and limited room for integration. With the following examples we bring about a discussion on the potential of complex photonic networks to implement natural computing.

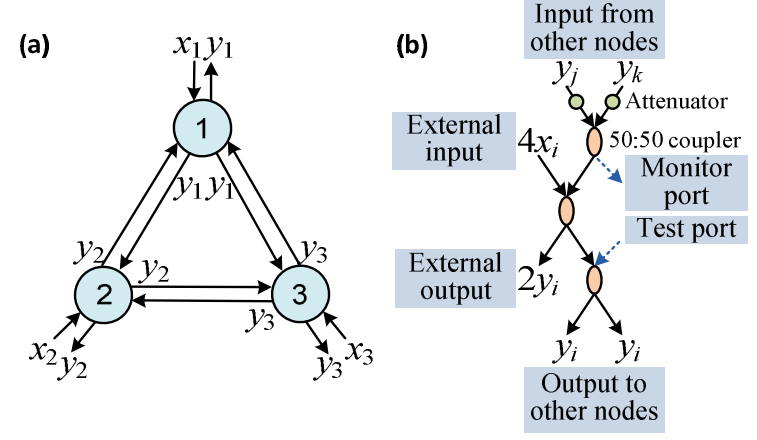

(c)

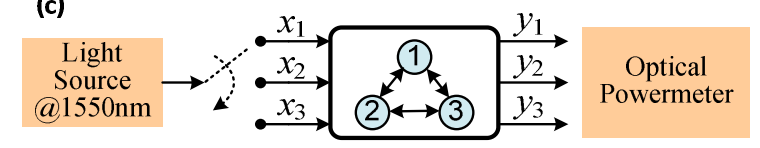

Figure 1: (a) Schematic of an optical fiber network with three nodes where $x_{i}$ and $y_{i}(i=1,2,3)$ are the input and output ports of each node; (b) Actual design of a node with optical fiber, couplers and attenuators and (c) Experimental setup of calculating matrix inversion.

\section{DISCUSSION}

\subsection{Linear optical networks for polynomial and nondeterministic polynomial problems}

We first demonstrate the possibility of using an optical fiber network to calculate inverse matrices
[1]. A $3 \times 3$ matrix is inverted as a proof-of-concept demonstration using a fiber network containing three nodes and operating at telecomm wavelength (Fig. 1). For an $N \mathrm{x} N$ matrix, the overall solving time (including setting time of the matrix elements and calculation time of inversion) scales as $\mathrm{O}\left(N^{2}\right)$, whereas matrix inversion by most advanced computer algorithms requires $\sim \mathrm{O}\left(N^{2.37}\right)$ computational time. For well-conditioned matrices, the error of the inversion performed optically is found to be around $3 \%$, limited by the accuracy of measurement equipment. Besides the experimental demonstration, potential and limits of this approach are also discussed, including extension to complex matrix elements, calculation precision and accuracy, and scalability. Applicability of this method to solve the eigenvalue problem is also proposed.
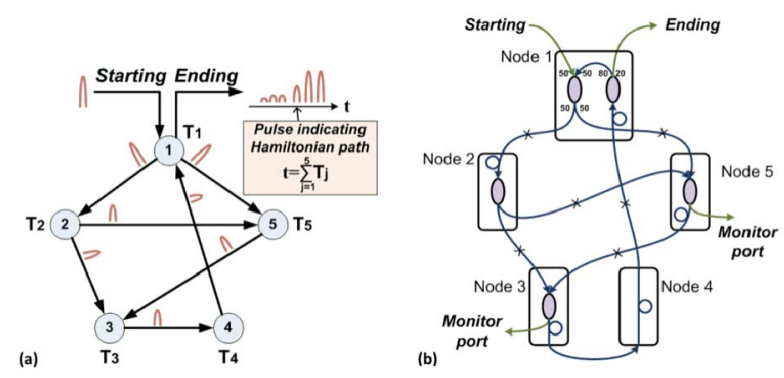

Figure 2: (a) "Optical oracle" for the solution of the Hamiltonian path problem on a target graph with five nodes. The Hamiltonian path exists if a pulse returning to node 1 is observed after a delay equal to the total delay of the whole network. (b) Actual design of the graph with optical fiber components.

Using a fiber network with 5 nodes (Fig. 2), we then provide a proof-of-principle demonstration that it can be seen as an optical oracle for the Hamiltonian path problem, the famous mathematical problem of finding if a map can be travelled so that each town is visited once only [2]. Pronouncement of a Hamiltonian path is made by monitoring the delay of the optical pulse interrogating the network, which shall be equal to the sum of travel times visiting all the nodes (towns). Although it does not break the limitation of exponential solving time for NPcomplete problems, our approach allows solving this NP-complete problem hundreds times faster than brute-force computing, and could be scaled to analyze maps of a considerable size. This may

\footnotetext{
${ }^{1}$ Centre for Disruptive Photonic Technologies, Nanyang Technological University, 21 Nanyang Link, Singapore 637371

${ }^{2}$ Optoelectronics Research Centre, University of Southampton, SO17 1BJ, UK

*corresponding author, e-mail: csoci@ntu.edu.sg
} 
already find application in optical routing and secure communications.

\subsection{Nonlinear optical networks for metaheuristic optimization}

Using nonlinear Erbium doped optical fiber network we implemented the famous ant colony optimization algorithm (ACO) for finding the shortest path on the graph that connects the ant colony to the foraging area [3]. ACO algorithms are inspired by the behavioral pattern of real ants, by which they are able to find the shortest path leading to food using indirect communication mediated by modifications of the environment (stigmergy). When they find food, ants exchange information by secreting volatile chemicals (pheromones) on their way back to the nest. The pheromone increases the probability that other foragers will follow the same path towards the food source, so that ants collectively develop a complex network of trails, connecting the nest to the foraging area in the most efficient way. This network of trails can be seen as a shared memory for the entire colony.

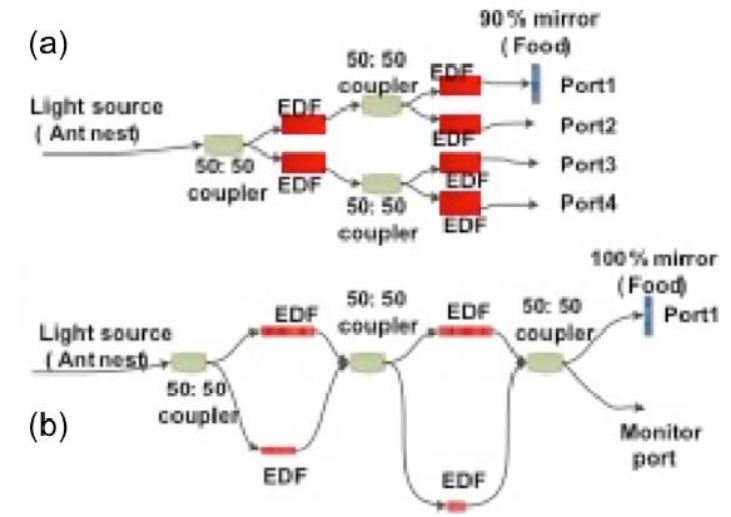

Figure 3: Network design schemes used to demonstrate all-optical ACO: (a) two-layer tree and (b) two-layer double bridge experiment.

In the nonlinear optical fiber network (Fig. 3), the optical power corresponds to the number of ants, transient saturable absorption of Erbium doped fibers mimics the function of pheromone, and an optical reflection mirror is used to represent the food. ACO is demonstrated in two typical design schemes: in a tree-like scheme (Fig. 1a), the path connecting food and nest accumulates the largest optical power (number of ants) compared to other paths, while in a double-bridge scheme where few alternative paths connect the nest to the food (Fig. 1b), the optical output signal from the shortest path is reinforced as a function of time and optical input power.

\subsection{Toward all-optical neuromorphic computing}

We finally show how amorphous chalcogenide microfibers of the alloy gallium lanthanum oxysulphide (GLSO) may be used as a platform to realize all-optical neuromorphic systems with neuronal axon and synapse functionalities (Fig. 4). As a last proof-of-concept, we demonstrate a variety of neurophysiological phenomena in the optical regime mimicking communication protocols in the mammalian central nervous system, including temporal and spatial summation, excitatory and inhibitory post-synaptic potentials, and short and long term plasticity [4].

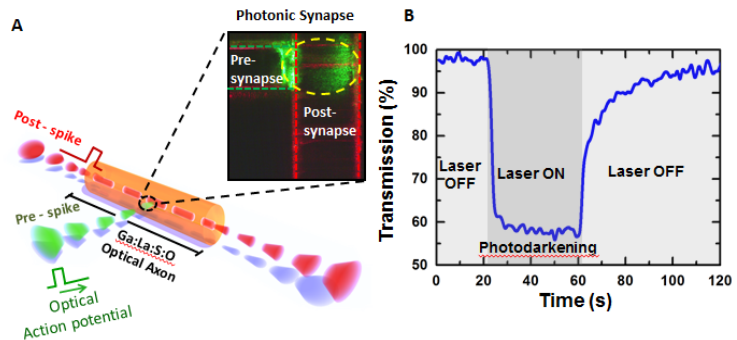

Figure 4: (a) The optical neuron transmits information through optical signals (laser pulses) that propagate along a gallium lanthanum oxysulphide fiber, and use photodarkening as a result of subbandgap light exposure at the photonic synapse (inset) to induce inhibitory and excitatory action potentials in the post-synaptic axon. (b) Photodarkening occurring upon exposure of the GLSO microfiber to sub-bandgap excitation reduces transmission of guided light by $\sim 40 \%$.

\section{CONCLUSIONS}

We provide few examples of natural computing tasks performed by optical fibre networks. This framework may be readily extended to integrated silicon photonics or plasmonic waveguide networks to tackle problems of greater complexity.

\section{Acknowledgements}

Research was supported by the Singapore Ministry of Education Academic Research Fund Tier 3 (Grant MOE2011-T3-1-005), and by the Singapore Agency for Science, Technology and Research (A*STAR, SERC Project No. 1223600007).

\section{References}

[1] K. Wu, C. Soci, P.P. Shum, N.I. Zheludev, Computing matrix inversion with optical networks, Opt. Express 22, 295-304 (2014).

[2] K. Wu, J. García de Abajo, C. Soci, P. P. Shum, and N. I. Zheludev, An optical fibre network oracle for NP-complete problems, Light: Science \& Applications 3, e147 (2014).

[3] W. Hu et al., to be submitted (2014).

[4] B. Gholipour et al., to be submitted (2014). 